\title{
Comparison of Finite Element and Analytic Calculations of the Resonant Modes and Frequencies of A Thick Shell Sphere
}

\author{
David E. Gilsinn \\ Hans J. Hansen* \\ Xu-Yanshen * * \\ Miguel Huerta-Garnica * * * \\ E. Clayton Teague
}

\author{
U.S. DEPANTMENT OF COMMERCE \\ Natlonel Institute of Standards \\ and Tochnoloty \\ Manufecturins Endinecind Leboratory \\ adthorburt, MB 20899

\footnotetext{
*Tencor Instrumonto

* Tianilin Univoraty,

Tlanila, PRic Averandos dol IPN, Moxleo
} \\ ** Contro do Investifsecion y de Estudios
}

Certain commorelal coftware and equipanont is identified in this paper in orden to edequately epecify procedure. Such identifiedton does not imphy reconmendation or endoreoment by the Nationel Institute of Standards and Technolosy, nor does it imply thet the software Identified is nocesearily the bost aveilable for the purpoce.

US. DPPATIM JT OF COMU zReE Robert A Modoche, Secret.y

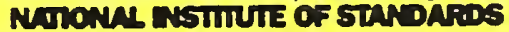
AS TECHNCLOCY John W Lyons, Dinector 



\title{
Comparison of Finite Element and Analytic Calculations of the Resonant Modes and Frequencies of A Thick Shell Sphere
}

\author{
David E. Gilsinn \\ Hans J. Hansen* \\ Xu-Yanshen** \\ Miguel Huerta-Garnica* * * \\ E. Clayton Teague
}

\section{U.S. DEPARTMENT OF COMMERCE \\ National Instrturte of Standards \\ and Toehnolocy \\ Manufacturins Endinoerins Leboratory \\ Odthorebur, MD 20899}

\author{
* Tencor Instruments \\ * Tianilin Univeraty, \\ Thenlin, Pric \\ - * Contro de Investifacion y de Estudios \\ Averzedos del IPN, Mexleo
}

Certaln commorclal coftwere and equiproat is Identified in this paper in onder to edequately epechy procedure. such identifiention does not Imply recomanendrtion or endorement by the Netionel invthite of Standards and Technoley, nor does it Imply thet the coftwere idemtined is necesently the bost avdlable for the purpose.

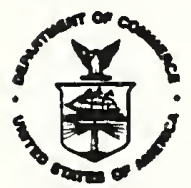





\section{Abstract}

An ultra-high accuracy planar coordinate measuring machine, called the Molecular Measuring Machine $\left(\mathrm{M}^{3}\right)$ is being built at the National Institute of Standards and Technology (NIST). The design goal is to achieve a spatial resolution of $0.1 \mathrm{~nm}$ over an area of $50 \mathrm{~mm} \times 50 \mathrm{~mm}$. This goal requires that gravity loading and vibration response of a complicated mechanical structure be modeled to a high accuracy. Finite element analysis (FEA) was applied in the design of $\mathrm{M}^{3}$ since analytical modeling of the complicated structure was precluded. In order to achieve confidence in a specific FEA package, normal mode analysis results for a thick spherical shell that represented a first approximation to the $\mathrm{M}^{3}$ structure were compared to those predicted by another FEA package and to analytic results of the thick shell problem. The analytic model predicted degenerate modes due to the structural symmetry of the sphere. These were confirmed subsequently by both of the FEA packages. The FEA and analytic results differ by less than 58 .

Keywords: boundary value problems; finite element analysis; Molecular Measuring Machine; normal mode analysis; software evaluation; thick shell sphere; vibration 


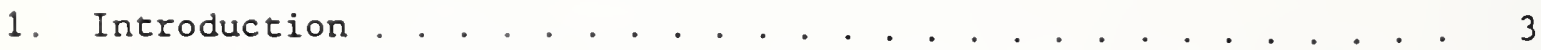

2. Measurement Machine Simulation................ . 3

3. Analytic Model ..................... . . 4

4. Finite Elements .. . . . . . . . . . . . . . . 5

5. Computation Concerns . . . . . . . . . . . . . . . 6

6. Simulating Boundary Conditions... . . . . . . . . . . . 6

7. Mesh Selection Strategy . . . . . . . . . . . . . 6

8. A Comparison with Analytic Results. . . . . . . . . . . . . 7

9. Conclusions . . . . . . . . . . . . . . . . . 8

10. References....................... . . . . 9

11. Appendix . . . . . . . . . . . . . . . . . 9

\section{Figures}

Figure 1. $\mathrm{M}^{3}$ Core Structure . . . . . . . . . . . . . . . 12

Figure 2. Sample Vibration Modes . . . . . . . . . . . . . . 13

Figure 3. Decomposition of a Region . . . . . . . . . . . . . . . . 14

Figure 4. Mesh Models . . . . . . . . . . . . . . . . 15

Figure 5. 192 Element Mesh Model Used by GEMINI . . . . . . . . . . 16

Figure 6. Sample Modes Computed by GEMINI at Three Frequencies . . . 17

Figure 7. First Three Modes Computed by COSMOS . . . . . . . . . . . 18

\section{Tables}

Table 1. Percentage Deviation of Finite Element Results... . . . 19

Table 2. Frequency Comparison . . . . . . . . . . . . . . 20 


\section{INTRODUCTION}

The National Institute of Standards and Technology (NIST) has embarked on a project to construct an instrument capable of providing very high resolution dimensional metrology information about topographic features for a wide variety of sample surfaces and materials. The design goal is to obtain a point-to-point spatial resolution of $0.1 \mathrm{~nm}$ of the distance between any two points within a $50 \mathrm{~mm} \times 50 \mathrm{~mm} \times 100 \mu \mathrm{m}$ working volume. The maximum size of the specimens will be limited to 50 $\mathrm{mm} \times 50 \mathrm{~mm} \times 12 \mathrm{~mm}$. Typical topographic surface maps will be 1000 pixels $\times 1000$ pixels with two modes of operation. The large scale or coarse mode of operation will allow acquisition of up to a $50 \mathrm{~mm} \times 50 \mathrm{~mm}$ image at resolutions of about $1 \mathrm{\mu m}$; the small or fine scale map will allow acquisition of a $100 \mathrm{~nm} \times 100 \mathrm{~nm}$ image at resolutions of less than $0.2 \mathrm{~nm}$. The probe-to-specimen speed is expected to be $1 \mathrm{~mm} / \mathrm{s}$ or less.

Due to the extreme accuracy demands of the instrument, relative displacements between the surface probe tip and the sample caused by gravity loading and vibration had to be estimated. The core structure of $\mathrm{M}^{3}$ is a complex spherical form, see Fig. 1, and precludes analytic estimates of the displacements. Finite element analysis (FEA) was selected as an appropriate technique to address the boundary value problems that need to be solved to estimate both gravity load deformations and vibration modes. Since finite element analysis was also going to be used in other aspects of the design of $\mathrm{M}^{3}$ it became essential to verify the results of a particular package. Results of the two software FEA packages were therefore tested against each other and against the results of an analytic model so that a confidence level could be established for the accuracy of the solutions to the thick shell problem. This would also give confidence in other applications of the finite element analysis method. This paper discusses our computational experience and how the FEA and analytic results compare for the simple thick shell sphere model. In some recent related work Tang, Fong, and Dietrich [1] have used new capabilities introduced in another FEA package that changed a comparative experiment, as given in this report into an active one in which a user not only could monitor errors for a benchwork problem but develop probes to measure error changes due to perturbations of mesh design. They demonstrated that these FEA features could be used to develop an automated mesh generation procedure that would make the FEA results approach the exact solution of their benchmark problem. They applied these features to the analysis of the stresses in the vicinity of an elliptical hole in a uniformly stressed plate. Since the data we obtained by the comparative technique were sufficient for our study and these active monitoring features were not available on our FEA package, this approach, although it showed promise, was not pursued.

\section{MEASUREMENT MACHINE SIMULATION}

Since the instrument core structure is basically a sphere with an inner core removed, $\mathrm{M}^{3}$ was modeled as a thick-shelled aluminum hollow sphere with an inner and outer radii of $10 \mathrm{~cm}$ and $20 \mathrm{~cm}$, respectively. 
Material properties were chosen for an aluminum alloy with a Poisson's ratio of 0.355 , modulus of elasticity of $71.3 \mathrm{GPa}\left(10.3 \mathrm{Mlb} / \mathrm{in}^{2}\right)$, unit weight of $26.6 \mathrm{kN} / \mathrm{m}^{3}\left(0.098 \mathrm{lb} / \mathrm{in}^{3}\right)$, and specific stiffness of $2.6 \times 10^{7} \mathrm{~m}^{2} / \mathrm{s}^{2}\left(4.04 \times 10^{10} \mathrm{in} / \mathrm{s}^{2}\right)$. At the time the calculations were performed, aluminum was thought to be the material of choice. A subsequent figure of merit analysis showed oxygen-free-high-conductivity copper to be the better material. The material properties used for copper were: Poisson's ratio of 0.326 , modulus of elasticity of $119 \mathrm{GPa}$ ( 17.2 $\left.\mathrm{Mlb} / \mathrm{in}^{2}\right)$, unit weight of $87.3 \mathrm{kN} / \mathrm{m}^{3}\left(0.322 \mathrm{lb} / \mathrm{in}^{3}\right)$, and specific stiffness of $1.3 \times 10^{7} \mathrm{~m}^{2} / \mathrm{s}^{2}\left(2.1 \times 10^{10} \mathrm{in}^{2} / \mathrm{s}^{2}\right)$. The frequencies for copper in Fig. 2 were estimated based on the formula relating frequencies and eigenvalues of the exact boundary value problem in the appendix in Section II. Since the Poisson's ratio of aluminum and copper are sufficiently close and the eigenvalues of the boundary value problem depend on the Poisson's ratio and inner and outer radii of the thick shelled sphere the eigenvalues in both material cases are nearly the same. The eigenvalue to frequency formula could be equated for the two materials and the frequencies for copper thus computed.

\section{ANALYTIC MODEL}

The equation of motion of any homogeneous, isotropic, elastic medium can be written in the form of a vector wave equation (See Appendix). The time dependent portion of the solution can be separated out in order to compute the normal modes of vibration and their frequencies. The result is a vector Helmholtz equation where the eigenvalues are related to the angular frequencies of the normal modes. The solution of this eigenvalue problem can be written in the form of a sum of three vector fields. These three vector fields are related to three scalar fields that are themselves solutions of three scalar Helmholtz equations with eigenvalues, which are determined by imposing boundary conditions. The solutions for these scalar equations can be found by separation of variables. In spherical coordinates the solutions can be written as products of radial functions and angular functions. The radial functions, indexed by $n$, can be written as linear combinations of spherical Bessel and Neumann functions. For each of these radial functions there are $2 n+1$ angular functions. These are spherical harmonics indexed by $n$ and another parameter $m$, where $m$ takes on the $2 n+1$ values from $-n$ to $n$.

Physically, each mode of vibration, indexed by $n$, has an $n$-fold symmetry. These modes arise without consideration of boundary conditions. Once the boundary conditions are imposed, the eigenvalues of the scalar Helmholtz equations can be determined. These are directly related to the eigenfrequencies of the overall vector Helmholtz equation. For the thickshelled sphere problem, stress free boundary conditions were assumed at the inner and outer surfaces since the $M^{3}$ will be operated in vacuum. From these conditions it can be shown that there are an infinite number of eigenfrequencies, indexed by $s$, for each mode $n$. Note that this means the same value of $s$ can have different frequency values depending on the mode $\mathrm{n}$. It can further be shown that the eigenfrequencies are independent of the index $m$ due to the symmetry of the sphere, resulting in a $2 n+1$ 
degeneracy of each $\mathrm{n}$-order mode.

Analytically, vibration modes of the thick-walled sphere decompose into two classes. In one class, there is no change in the volume of the sphere and radial displacements are zero. These are referred to as torsional modes. In the second class, radial displacements are not zero. These modes, termed spheroidal vibrations, were the only ones considered in the analytical solution since they were considered to have the greatest impact on the performance of the $M^{3}$. For more details on the analytical solutions for torsional modes of a solid sphere see Hosseini-Hashemi and Anderson [2] and for radial modes of a spherical shell see Mehl [3] and Huerta-Garnica and Teague [4]. Huerta-Garnica and Teague obtained the radial modes for larger shell thicknesses than Mehl and calculated the dynamic response for point excitations of the sphere.

Figure 2 shows 4 sample modes from the analytical solutions. In general, the analytical modes are specified by three indices, $n, m, s$, where for each $n$ and $s$ there are $2 n+1$ different modes with the same frequency. For all of the examples provided, $s$ is equal to 1 while $n$ and $m$ vary. Using the $(n, m, s)$ notation, the $(0,0,1)$ mode is a "breathing" mode where the inner and outer surfaces move in phase radially. The $(1,0,1)$ mode may be thought of as an "egg yolk" mode, where the inner and outer surfaces move out of phase with respect to one another. In the $(2,1,1)$ mode all points move radially with the inner and outer surfaces moving in the same direction to form an elliptically shaped mode. The final mode illustrated has a triangular shape.

\section{FINITE ELFMENTS}

The finite element method approximates a solution of a boundary value problem by a set of piecewise differentiable functions, each one of which is defined over a finite number of subregions that combine to form the total region over which solution is defined. The subregions are called elements and are defined by sets of points called nodes (see Fig. 3). The displacement values at the nodes of the solution of the boundary value problem are called the degrees-of-freedom and are the quantities to be computed. The piecewise differentiable functions selected as approximations to the solution over each element are sums of polynomials defined in terms of the nodal degrees-of-freedom. These polynomials are called shape functions and are chosen to maintain continuity at the interelement boundaries. The nodal degrees-of-freedom for each element are solved for as solutions of linear equations whose coefficients involve integrals of the boundary value problem operators applied to the shape functions. These are combined using the connectivity of the elements to form a large matrix called the stiffness matrix, [K]. The Jth column of [K] can be viewed as the forces needed to maintain static equilibrium with a unit displacement of the Jth degree-of-freedom. For the free vibration frequencies $\omega$ and modes one needs to solve the eigenvalue problem

$$
\left([K]-\omega^{2}[M]\right)(D)=\{0\} \text {, }
$$


where $[M]$ is known as the consistent mass matrix and is formed from integrals involving the mass density $\rho$ and the shape functions. The vector (D) represents the modal shape vector.

\section{COMPUTATION CONCERNS}

Several issues arose during the finite element analysis of the thickshelled sphere. The first problem involved determining how to model the boundary conditions with the data input languages provided by the two finite element packages used. The next issue was how to select a finite element mesh that would serve to compute the modes of interest. The third concern was how well the results would agree with those from the analytic solution. A final issue was how well the results from different finite element programs would agree.

In order to compare results from different programs, identical problems were analyzed with two different programs running on two different computers. One program was COSMOS/M from Structural Research and Analysis Corporation (SRAC); the other program GEMINI was developed at Lawrence Livermore National Laboratory (LLNL). COSMOS was run on a SUN Microsystems 3/260, while GEMINI was run on a VAX $11 / 785$.

\section{SIMULATING BOUNDARY CONDITIONS}

The model was not constrained in any of the three translational degrees-of-freedom in order to simulate the free-free case. Rotational degrees-of-freedom were constrained since the 3-dimensional solid finite elements available in the FEA packages had no rotational stiffness defined.

\section{MESH SELECTION STRATEGY}

The issue of mesh geometry became interwoven with the issues of comparisons between analytic solutions and finite element solutions. In general, various mesh geometries were selected differing primarily in the number of elements used. For the COSMOS system, five meshes were considered, but on the GEMINI system only two were used due to reduced computer time available on the VAX 11/785. In all cases 8 -node solid elements were used. These elements are forms of generalized hexahedrons. The inner and outer radii and material properties of the spherical shell were taken to be the same in both the analytic and the finite element models.

Although the specific mesh generation techniques for Cosmos and GEMINI were different certain guiding principles were used. The first guiding principle used with both packages was to select solid elements so that the inner and outer surfaces of the shell were covered with element surfaces that had approximately the same surface area. For the case of COSMOS this is shown in Fig. 4 for five different meshes. The final mesh 
selection depended on a convergence computation discussed below. In the GEMINI package two meshes were studied, one for 192 elements and one for 384. Fig. 5 shows the 192 element mesh for GEMINI.

\section{A COMPARISON WITH ANALYTIC RESULTS}

Table 1 gives a subset of frequency results of the analysis performed with the finite element packages and provides a comparison with the analytical results. The basic problem posed to the finite element codes was to determine the first 19 modes for a freely vibrating hollow sphere. The table lists the first two computed spheroidal frequencies and gives percent difference using the analytical results as a base. Some solution times are also provided. Torsional modes predicted by the finite element analysis are not indicated since the analytical method, by design, did not produce any of these modes.

These results indicate variations with both mesh density and with the FEA program used. In the case of mesh density, one would expect that with insufficient elements, accuracy for the higher modes would suffer due to insufficient discretization. On the other hand, high mesh density can decrease accuracy due to roundoff, with an added penalty of increased solution time. A possible heuristic method for selecting mesh density is to compare results for different densities and select the model with a density such that it produces the minimum change in results from increasing or decreasing the density. It appears from Table 1 that a mesh density of around 192 elements is reasonable for this problem.

Comparing the results of the two codes (Table 2) shows that the differences between computed frequencies is less than 5z. Mode shapes from the two programs for models of the same density were virtually identical for the first 10 modes. Figure 6 shows sample modes computed with GEMINI. These can be compared to the corresponding modes computed analytically, as shown in Figure 2. Table 2 shows radial analytic modes aligned with comparable radial modes generated by the finite element programs. Note that the finite element programs also produced torsional modes in groups that are interleaved with the radial modes. Due to symmetry they also have the same degeneracies as the radial modes. This table also shows that GEMINI produced consistently lower frequency values than the analytic results, while COSMOS produced consistently higher results. This is attributable to different computer algorithms for generating the frequencies.

Although mesh density differences are studied here other factors might account for some of the differences in the results. Both integration procedures used and the choice of shape functions by the different packages can produce different results. However it is usually not possible for the analyst to change these in the package since these are built into the element definitions provided by the FEA package supplier. The present study, then, has concentrated on studying variations in those parameters that are available to the FEA package user. 
Table 2 shows the first 19 frequencies of the non-rigid body modes organized into groups as computed by COSMOS and GEMINI. The first five modes represent the lowest frequency radial mode with its five-fold degeneracy. Note that neither finite element program produces the same frequencies for these modes the way the analytic solution does. This points out the need for using some analytic methods in conjunction with finite element tools as an interpretive guide in the case of structural symmetries. Without the analytic knowledge of the degeneracy property it might have been difficult placing the finite element modes in their proper groups. For example in the first ten modes note that both finite element programs produce frequencies grouped in two groups of two and two groups of three. Modes 1 and 2 could be isolated as a separate group from modes $3,4,5$ without an analytic guide.

Figure 7 shows typical output from the first three modes generated by COSMOS as given in Table 2. Pictures of all of the degeneracies are not shown. The figure shows that the mode computed at $5918 \mathrm{~Hz}$ is clearly a torsional mode. This mode was not computed analytically.

\section{CONCLUSIONS}

Comparing finite element and analytical results showed that in general the frequencies computed with the finite element method agreed well with corresponding analytical results. Two explanations for differences are suggested. One is that results from the analysis of a perfectly symmetrical object, (i.e., a hollow sphere) are affected by the artificial stiffness introduced by discretization. Another is that discretized representations only approach continuous ones in the limit as the number of elements becomes large. Unfortunately, increasing the number of elements leads to roundoff-born inaccuracies as well as longer solution times.

This study exposed certain practical observations about testing software in particular finite element packages. Since real life structures may be too complicated to model analytically a good rule seems to be to select a structure that can be modeled analytically but exhibits a structure as close as possible to the desired structure. This gives an analytic guide to the finite element analysis of the real structure. Another observation is that finite element packages that might be tested against each other may not be available on the same computer. This might lead to arithmetic differences in the results. Finally different packages construct meshes differently and use different algorithms for solving the eigenvalue problems that result. This suggests that the different FEA packages might be tested using standard eigenvalue problems with known solutions. However, the two packages tested do not provide a means of entering a user specified eigenvalue problem independent from one generated through a meshed model, which further suggests that a set of reference problems that probe the performances of an FEA package would be useful. 
Precision designing with FEA packages demands as much care in determining the operating characteristics of the software packages as one takes in determining the operating characteristics of precision laboratory instrumentation.

\section{REFERENCES}

1) Tang, J., Fong, J.T., Dietrich, D.E., "A Built-In Error Estimator for Optimizing Finite Element Modeling," ASME PVP Conf., Honolulu, HI July $23-27,1989$.

2) Hosseini-Hashemi S., Anderson J.S. "Orthogonality and Normalization of Torsional Modes of Vibration of Solid Elastic Spheres", J. of Sound and Vibration (1988) 121 (3), 511-524.

3) Mehl J.B., "Spherical Acoustic Resonator: Effects of Shell Motion", J. Acoust. Soc. Am. 78 (2) August 1985, 782-788.

4) Huerta-Garnica M.A and Teague E.C., "Frequency Response of a Thick Spherical Shell to a Local Surface Excitation", to be published.

\section{APPENDIX}

The displacement vector $\vec{u}(\vec{r}, t)$ for the motion of any homogeneous, isotropic, elastic medium can be written formally as

$$
c_{\ell}^{2} \vec{\nabla}(\vec{\nabla} \cdot \vec{u})-c_{t}^{2} \vec{\nabla} \times \vec{\nabla} \times \vec{u}=\partial^{2} \vec{u} / \partial t^{2}
$$

where $C_{\ell}$ and $C_{t}$ are the longitudinal and transverse wave speeds. They are related to material properties of the medium by Young's modulus $E$ and Poisson's ratio $\sigma$ and density $\rho$

$$
\begin{gathered}
C_{\ell}^{2}=E(1-\sigma) /(\rho(1+\sigma)(1-2 \sigma)), \\
C_{t}^{2}=E /(2(1+\sigma) \rho)
\end{gathered}
$$

The solution of the displacement vector system can be found by separation of variables into a time-dependent and a steady-state portion. The time independent equation can be written in the form of an eigenvalue problem

$$
c_{\ell}^{2} \vec{\nabla}(\vec{\nabla} \cdot \vec{u})-c_{t}^{2} \vec{\nabla} \times \vec{\nabla} \times \vec{u}=-\omega^{2} \vec{u}
$$

The solution can be written in the form of a sum of a longitudinal component, $\vec{L}$, and two transverse components, $\vec{R}$ and $\vec{N}$, which may be represented in terms of three scalar fields, F, G and $H$,

$$
\vec{u}(\vec{r})=\vec{L}+\vec{M}+\vec{N}
$$


where

$$
\begin{aligned}
& k_{\ell} \vec{L}=\vec{\nabla} F(\vec{r}) \\
& \vec{M}=\vec{\nabla} \times(\hat{\operatorname{rrg}(\vec{r}))} \\
& \vec{k}_{t} \vec{N}=\vec{\nabla} \times \vec{\nabla} \times(\hat{\operatorname{rrH}}(\vec{r})),
\end{aligned}
$$

where $k_{\ell}=\omega / C_{l}, \quad k_{t}=\omega / C_{t}$. In these equations $\vec{r}$ represents the position vector of a point, $r$ its magnitude and $\hat{r}$ the unit radial vector at that point. The scalar fields defined above are solutions of the scalar Helmholtz equation and can be used to generate a general solution. The Helmholtz equations are:

$$
\left(\nabla^{2}+k_{l}^{2}\right) F(\vec{r})=0 ; \quad\left(\nabla^{2}+k_{t}^{2}\right) G(\vec{r})=0 ; \quad\left(\nabla^{2}+k_{t}^{2}\right) H(\vec{r})=0 .
$$

In spherical coordinates, these fields are linear combinations of spherical Bessel and Neumann functions for the radial part and spherical harmonics for the angular parts.

$$
\begin{array}{lll}
F(\vec{r})=F_{n}(r) Y_{n m}(\theta, \phi) ; & F_{n}(r)=B_{1} j_{n}\left(k_{l} r\right)+B_{2} y_{n}\left(k_{l} r\right) \\
H(\vec{r})=H_{n}(r) Y_{n m}(\theta, \phi) ; & H_{n}(r)=B_{3} j_{n}\left(k_{t} r\right)+B_{4} y_{n}\left(k_{t} r\right) \\
G(\vec{r})=G_{n}(r) Y_{n m}(\theta, \phi) ; & G_{n}(r)=B_{5} j_{n}\left(k_{t} r\right)+B_{6} y_{n}\left(k_{t} r\right)
\end{array}
$$

Finally, the displacement vector is given by:

$$
\begin{aligned}
\vec{u}(\vec{r})= & \hat{r}\left(\frac{d F_{n}}{d\left(k_{\ell} r\right)}+n(n+1) \frac{H_{n}}{k_{t^{r}}}\right) Y_{n m}(\theta, \phi) \\
& +\hat{\theta}\left(\left(\frac{F_{n}}{k_{\ell} r}+\frac{1}{k_{t} r} \frac{d}{d r}\left(r H_{n}\right)\right) \frac{\partial}{\partial \theta} Y_{n m}(\theta, \phi)+\frac{G_{n}}{\sin \theta} \frac{\partial}{\partial \phi} Y_{n m}(\theta, \phi)\right)
\end{aligned}
$$




$$
+\hat{\phi}\left(\left(\frac{F}{k_{\ell} r}+\frac{1}{k_{t} r} \frac{d}{d r}\left(r H_{n}\right)\right) \frac{1}{\sin \theta} \frac{\partial}{\partial \phi} Y_{n m}(\theta, \phi)-G_{n} \frac{\partial}{\partial \theta} Y_{n m}(\theta, \phi)\right)
$$

For an unconstrained shell in vacuum, we require stress free boundary conditions at $r=a$ and $b$,

$$
\sigma_{r r}=\sigma_{r \theta}=\sigma_{r \phi}=0 \text { at } \quad r=a, b
$$

where $a$ and $b$ are the respective inner and outer radii of the thickshelled sphere. These boundary conditions yield a homogeneous set of constraint equations for the $B_{i}, i=1, \ldots, 6$, in the defining equations for $F_{n}, H_{n}$, and $G_{n}$ above. The eigenvalues, $\lambda_{n s}$, of this system determine the resonant frequencies. The eigenvalues are functions of the ratio b/a and the Poisson's ratio $\sigma$ and are related to the frequencies by

$$
\lambda_{\mathrm{ns}}=2 \pi \mathrm{f}\left[\frac{(1-2 \sigma)(1+\sigma) \rho}{(1-\sigma) \mathrm{E}}\right]^{1 / 2} \mathrm{a},
$$

where f represents a resonant frequency. 


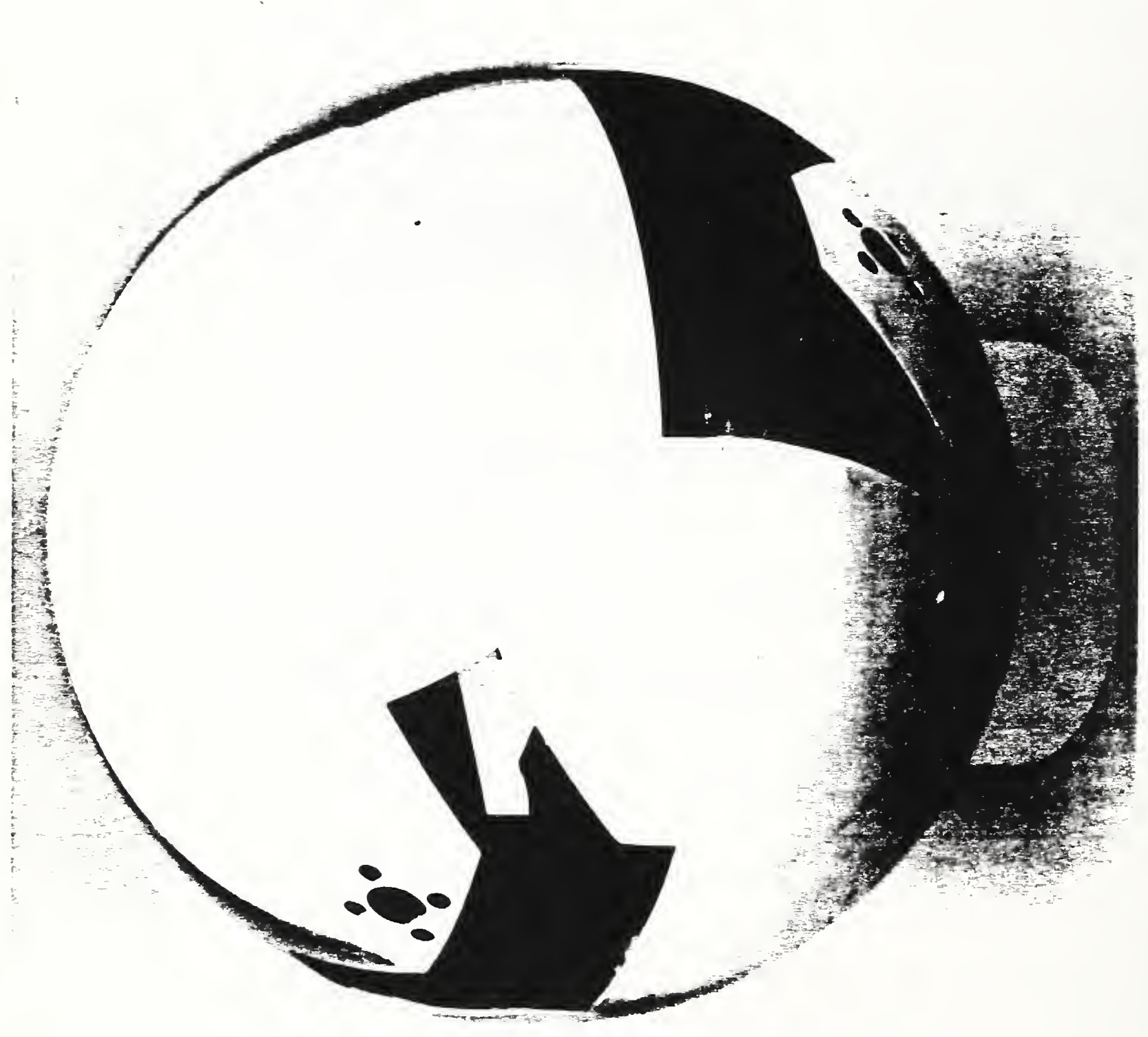

$\stackrel{0}{2}$

๖్

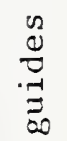

:

$=$

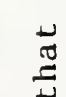

.

0
2
3
0
0
0
0
0
0
0
0
0
0
0
0
0
0
0

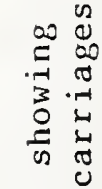

竎

的需

政

范

$-$

$\dot{0}$ 


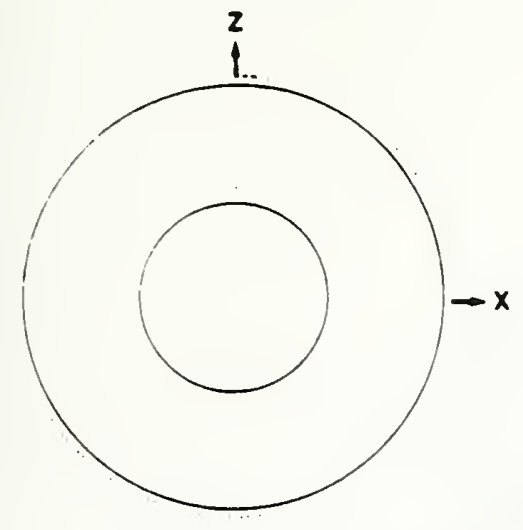

$\mathrm{n}=0, \mathrm{~m}=0, \mathrm{~s}=1$

Frequency

Aluminum $9994 \mathrm{~Hz}$ Copper $7039 \mathrm{~Hz}$

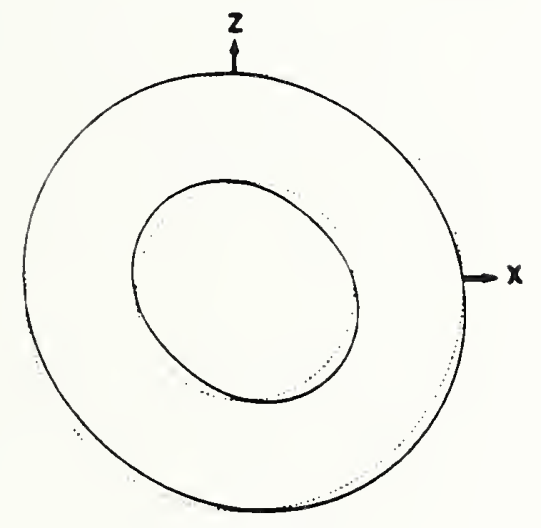

$n=2, \quad m=1, \quad s=1$

Frequency

Aluminum $4752 \mathrm{~Hz}$

Copper $3347 \mathrm{~Hz}$

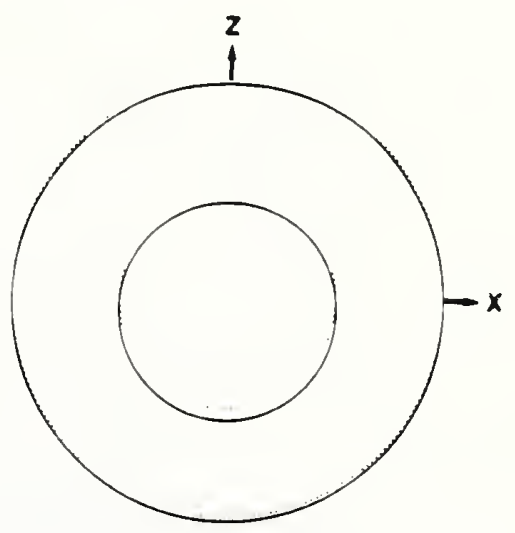

$\mathrm{n}=1, \mathrm{~m}=0, \mathrm{~s}=1$

\section{Frequency}

Aluminum $9944 \mathrm{~Hz}$ Copper $\quad 7004 \mathrm{~Hz}$

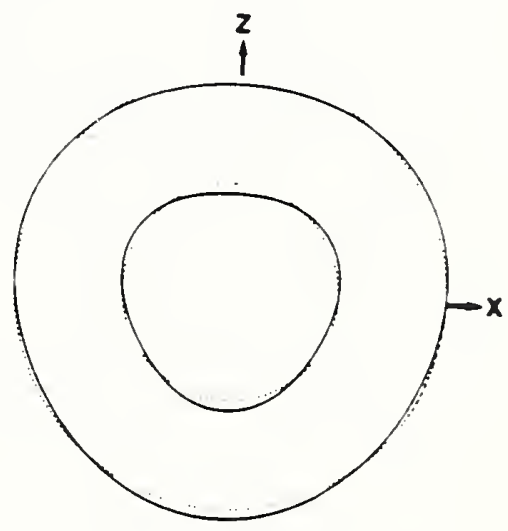

$\mathrm{n}=3, \mathrm{~m}=0, \quad \mathrm{~s}=1$

Frequency

Aluminum $7761 \mathrm{~Hz}$

Copper $5466 \mathrm{~Hz}$

Fig. 2 Sample vibration modes exhibited by the analytic solutions. 


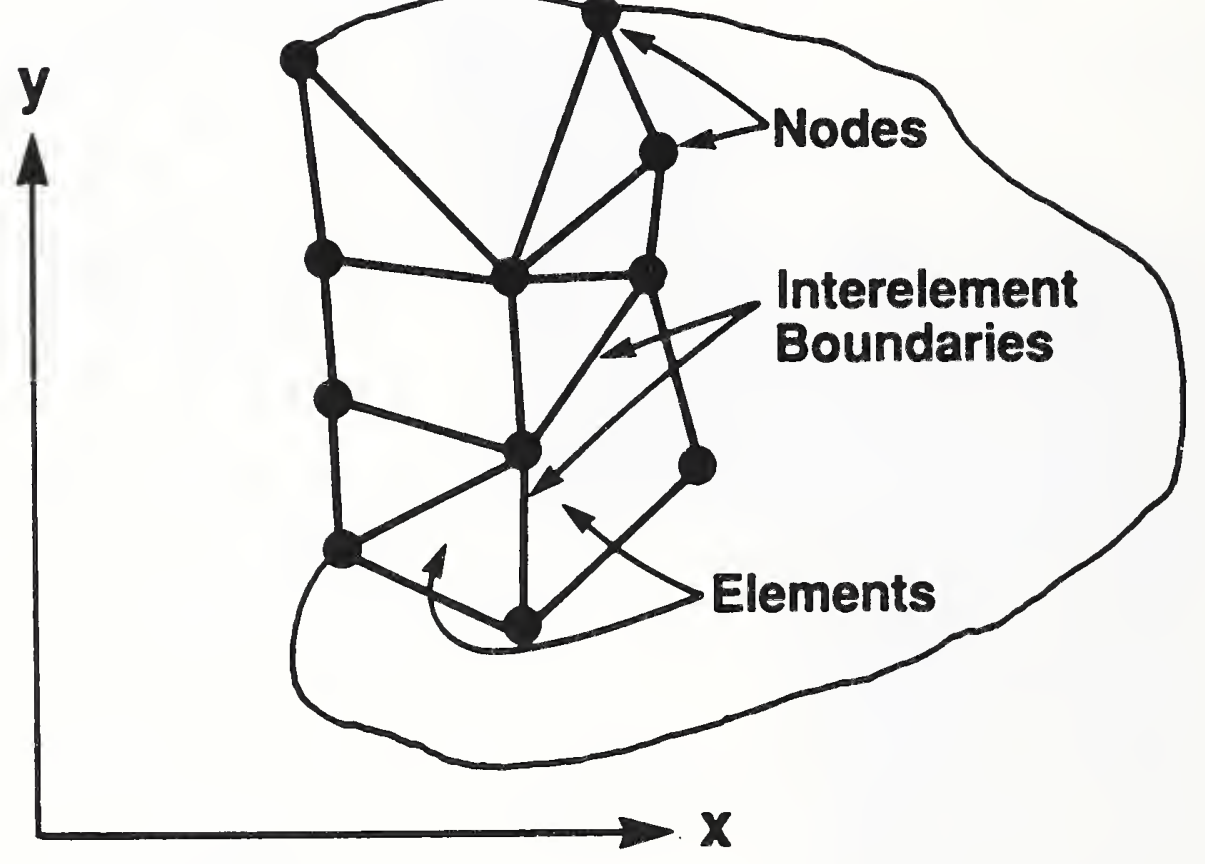

Fig. 3 Decomposition of a region for finite element analysis. 


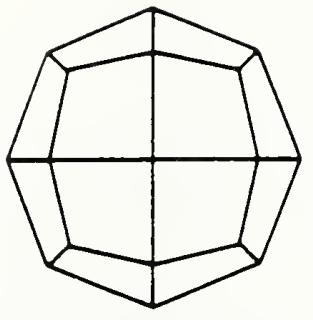

24

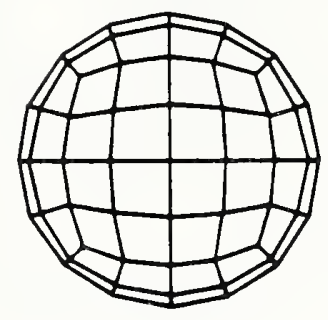

96

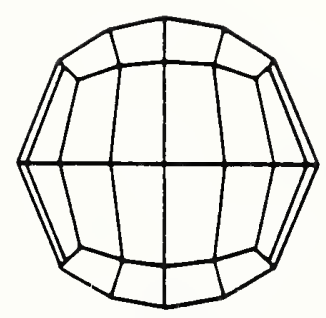

48

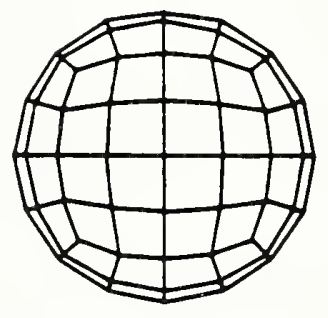

192

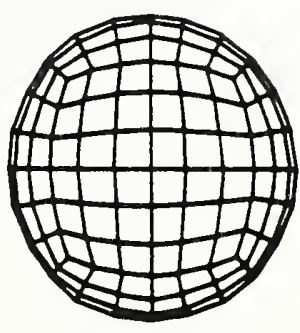

432

Fig. 4 Mesh models selected using elements with equal surface areas. Numbers represent total number of elements in the model. 


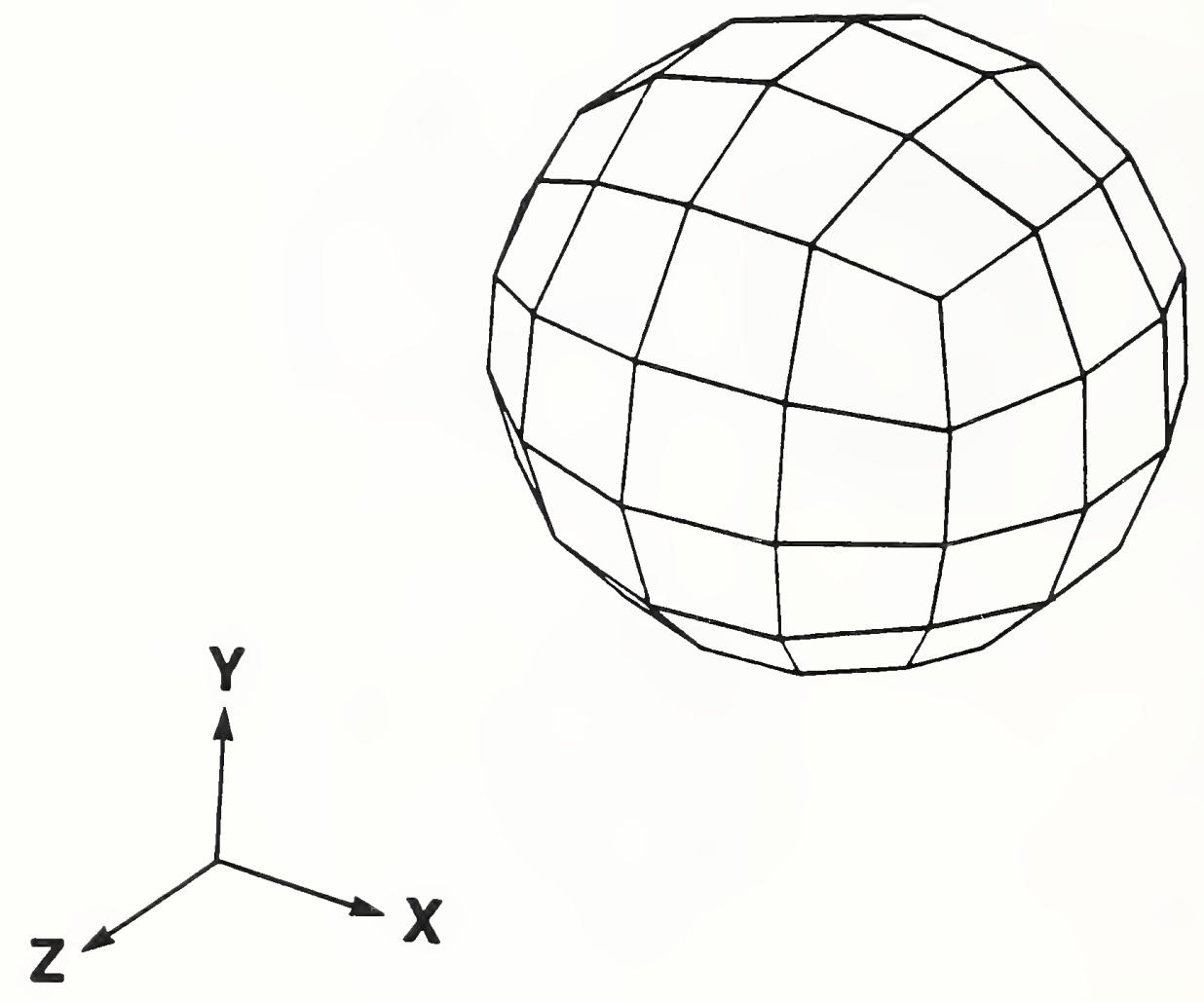

Fig. 5192 Element mesh model used by GEMINI. 


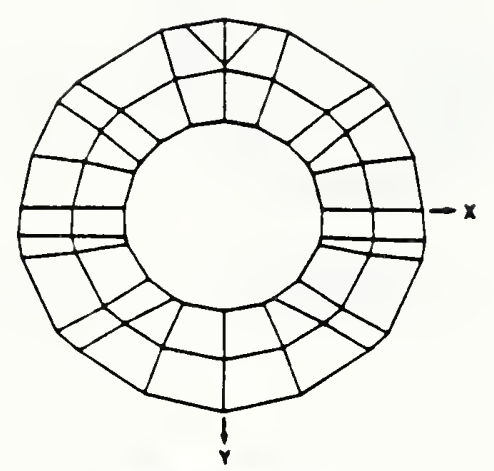

Frequency $8207 \mathrm{~Hz}$

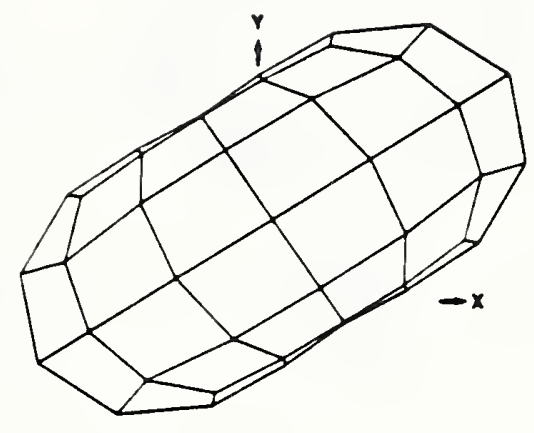

Frequency $4745 \mathrm{~Hz}$

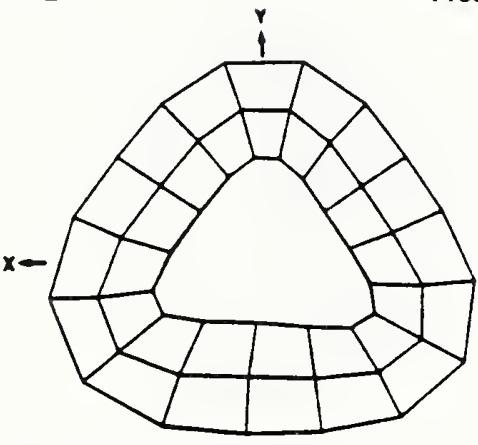

Frequency $7540 \mathrm{~Hz}$

Fig. 6 Sample modes computed by GEMINI at three frequencies. 


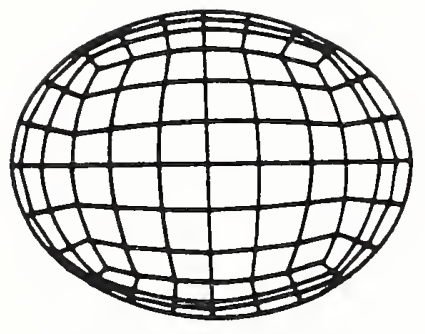

$4906 \mathrm{~Hz}$

Radial

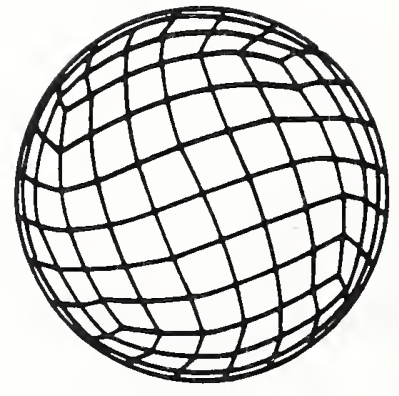

$5918 \mathrm{~Hz}$

Torsional

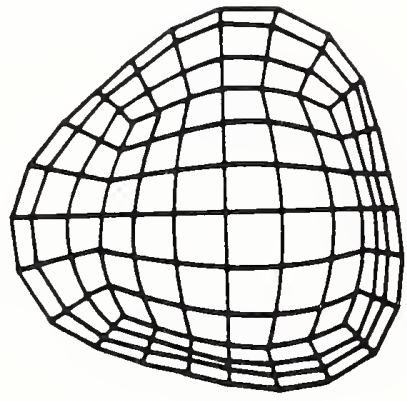

$7954 \mathrm{~Hz}$

Radial

Fig. 7 First three modes computed by COSMOS. Degeneracies are not shown. 
Table 1 Percentage Deviation of finite element results from analytical results.

Freq. (Hz) \&Difference Freq. (Hz) \&Difference Time (sec)

\begin{tabular}{|c|c|c|c|c|c|}
\hline $\begin{array}{l}\text { Analytic } \\
\text { COSMOS/M } \\
\text { (\#Elem.) }\end{array}$ & 4752 & & 7762 & & \\
\hline $\begin{array}{r}24 \\
48 \\
96 \\
192 \\
432\end{array}$ & $\begin{array}{l}5073 \\
4661 \\
4728 \\
4864 \\
4820\end{array}$ & $\begin{array}{r}6.8 \\
-2.0 \\
-0.5 \\
2.3 \\
1.4\end{array}$ & $\begin{array}{l}6970 \\
6931 \\
7520 \\
7807 \\
7854\end{array}$ & $\begin{array}{r}-10.0 \\
-10.7 \\
-\quad 3.7 \\
0.6 \\
1.2\end{array}$ & $\begin{array}{r}612 \\
1901 \\
3158 \\
5552 \\
37711\end{array}$ \\
\hline \multicolumn{6}{|l|}{$\begin{array}{l}\text { GEMINI } \\
\text { (\#Elem.) }\end{array}$} \\
\hline $\begin{array}{l}192 \\
384\end{array}$ & $\begin{array}{l}4744 \\
4738\end{array}$ & $\begin{array}{l}-0.2 \\
-0.3\end{array}$ & $\begin{array}{l}7540 \\
7538\end{array}$ & $\begin{array}{l}-2.9 \\
-\quad 2.9\end{array}$ & $\begin{array}{c}\text { NOT } \\
\text { AVAILABLE }\end{array}$ \\
\hline
\end{tabular}


Table 2 Mode grouping of the first 19 non-zero frequencies. Analytic frequencies in parentheses were not actually computed but would have resulted due to formula symmetry.

\section{FREQUENCY COMPARISON}

$\begin{array}{cc}\text { Mode } & \text { GEMINI } \\ \text { number } & \text { frequency } \\ & \text { (Hertz) } \\ & 192 \text { Elements }\end{array}$

ANALYTICAL

frequency

(Hertz)

COSMOS

frequency

(Hertz)

192 Elements

$\left.\begin{array}{rrrr}1 & & & \\ 2 & 4744.3 & 4751.9 & 4906 \\ 3 & 4744.4 & 4751.9 & 4906 \\ 4 & 4780.3 & (4751.9) & 4864 \\ 5 & 4780.4 & (4751.9) & 4864 \\ & 4780.6 & (4751.9) & 4864\end{array}\right\} \mathrm{n}=2,2 \mathrm{n}+1=5$



- 
